\title{
An Empirical Research on Influence of Users' Using Willingness of Internet Rumor-refuting Platforms
}

\author{
Teng jie $e^{1, a, *}$, xia zhijie ${ }^{1, b}$, teng qi $^{2, c}$ \\ ${ }^{1}$ Shanghai University of Engineering Science, Shanghai, China, \\ ${ }^{2}$ Nanjing normal University, Nanjing, China \\ adatengtengg@163.com, bxia_zhijie@163.com, cteqi159@gmail.com
}

\begin{abstract}
Keywords: internet rumor-refuting platforms, using intention, structural equation model, user experience
\end{abstract}

\begin{abstract}
Internet rumor-refuting platforms have made a step forward in combating rumors. But it lacks the attention of the audience participation. Therefore, internet rumor-refuting platforms' traffic and utilization rate are low. It is of great significance for the designers to improve the willingness to use the platform. This paper introduces user experience, perceived personal income and functional satisfaction, processes data through structural equation model, uses partial least square method, and verifies the model by measuring model and structural model.The research shows that perceived personal income and satisfaction directly affect the willingness to use the Internet rumour-refuting platform, and the functional satisfaction has a significant effect on satisfaction. Meanwhile, the weight of social experience about perceived personal income is second only to the utilitarian experience, which indicating that users are also satisfied with the social interaction of the platform while meeting the information needs.
\end{abstract}

\section{互联网辟谣平台用户使用意愿的实证研究}

\author{
滕婕 $1, \mathrm{a},{ }^{*}$,夏志杰 ${ }^{1, b}$,滕起 ${ }^{2, c}$ \\ 1 上海工程技术大学管理学院, 上海, 中国 \\ 2 南京师范大学电气与自动化学院, 南京, 中国 \\ adatengtengg@163.com, bxia_zhijie@163.com, cteqi159@gmail.com
}

关键词：互联网辟谣平台；使用意愿；结构方程模型；用户体验

摘要：互联网辟谣平台的建立固然使打击谣言工作向前迈进了一步，但平台的设计在受众的 存在感和参与体验感方面缺乏重视，导致平台使用率和访问量低等问题，因此，提高公众对 于互联网辟谣平台的使用意愿显得尤为重要。本文从用户使用意愿的角度，引入用户体验、 感知个人收益和功能满足度, 建立互联网辟谣平台用户使用意愿结构方程模型, 采用偏最小 二乘法对数据进行处理，并在测量模型和结构模型两个方面进行模型的验证。结果显示，感 知个人收益和满意度直接影响互联网辟谣平台用户使用意愿; 用户功能满足度对满意度的作 用显著; 社交体验对感知个人收益影响权重仅次于功利体验, 表明用户在满足信息需求的同 时，较为重视平台的社交互动性。

\section{1. 引言}

随着互联网的发展和普及程度的提高, 网络在很大程度上降低了人们获取内容的门槛, 提升了信息的传播效率，但也为谣言的生产与传播提供了更多的便利条件。谣言的传播污染 
网络环境、扰乱社会秩序, 严重损害了互联网的形象和公信力。为了减少谣言的负面影响, 已有研究从辟谣网站建设 ${ }^{[1]}$ 和政府管理 ${ }^{[2]}$ 的角度给出了提升互联网辟谣效果的建议。

互联网辟谣平台根本价值的实现来源于用户的采纳和广泛性使用。但在实践中，互联网 辟谣平台的使用率并不高，不同辟谣信息网站受众参与度差。为了鼓励更多的公众使用互联 网辟谣平台, 影响公众是否愿意使用互联网辟谣平台的因素研究非常重要。已有研究讨论了 一般信息系统平台的使用意愿问题，如社交平台 ${ }^{[3]}$ 、学习型系统 ${ }^{[4]}$ 等。但互联网辟谣平台与一 般的信息系统平台有较大的不同，这些因素对互联网辟谣平台是否同样有效目前还缺乏特别 的讨论。为弥补这些研究的不足, 本研究将在经典理论和大量文献分析的基础上提出若干假 设, 并进行实验收集实证数据, 识别影响用户使用互联网辟谣平台的关键因素, 据此为互联 网辟谣平台相关部门更好地利用平台、阻止谣言泛滥提供建议。

\section{2. 理论基础与研究假设}

当用户从平台中获得个人收益, 此处的个人收益无论功利收益, 利他收益和社交收益的 任何一种, 这都将刺激他们在互联网辟谣平台里进行在线内容的使用。Khan 等 ${ }^{[5]}$ 认为消费者 感知利益显著影响重购意愿。满意度是用户使用系统后的整体感受和评价, 这种感受和评价 是预测网络使用意愿的重要指标。另一方面, 当用户感知到互联网辟谣平台的使用增加了自 我收益时，用户的满意度会得以提升。因此，本研究提出研究假设：

假设 H1：互联网辟谣平台用户感知个人收益正向影响用户的使用意愿。

假设 H2：互联网辟谣平台用户满意度正向影响用户使用意愿

假设 H3：互联网辟谣平台用户感知个人收益正向影响用户的满意度

功利体验（Utilitarian Experience）是用户通过辟谣平台获得的资讯、知识等对自身有价 值的信息和帮助。当用户在平台上满足了信息需求, 功利性体验的获得会增加用户的收益 ${ }^{[6]}$, 如果用户认为, 在平台获得的功利体验是有价值的, 他对此平台的满意度也会提升。虚拟社 区的功利性体验会积极影响用户的满意度。因此, 本文提出研究假设 4 和假设 5:

假设 H4：互联网辟谣平台的功利体验正向影响用户的感知个人收益

假设 H5：互联网辟谣平台的功利体验正向影响用户的满意度

社交体验（Social Experience）是用户在参与辟谣平台活动时，从其他成员那里获得的亲 情、友谊和社会支持的体验。随着社会化媒体的发展，在线内容平台与社交类平台逐步融合， 用户从中体验到社交功能, 在享受平台所提供的内容服务中也能与其他用户进行交流和沟通 [7]。社交需求激励用户在辟谣平台上建立虚拟的人脉圈、搭建沟通交流的关系，以此满足用 户的交和归属感需要，用户的个人收益由此增加。当用户社交体验的满足会增加对互联网辟 谣平台的满意度, 因此, 本文提出如下假设:

假设 H6：互联网辟谣平台的社交体验正向影响用户的感知个人收益

假设 H7：互联网辟谣平台的社交体验正向影响用户的满意度

利他体验（Altruistic Experience）是指参与辟谣平台可以进行信息分享、信息传播等，通 过信息分享和传播帮助别人。与功利体验、社交体验属于利已型体验相反，利他体验是用户 在社区因帮助他人的行为而自身体验到的精神愉悦。用户通过分享、转发、评论辟谣信息的 行为帮助他人。利他性有助于用户树立正面的社会身份认知。因此, 本文提出如下的研究假 设:

假设 H8：互联网辟谣平台的利他体验正向影响用户的感知个人收益

假设 H9：互联网辟谣平台的利他体验正向影响用户的满意度

功能满足（Functional Satisfaction）反映的是，用户使用某一新媒体是因为新媒体在功能 上比传统媒体更能满足自己的某些需求。结合基于期望确认理论的信息系统持续使用模型的 理论逻辑, 用户使用后感知到的功能性满足对满意度有正向影响.不同的辟谣平台在信息质 量、系统设置等功能上都会存在差别或各有侧重, 从而给用户带来不一样的整体感受和满意 
度。所以, 本研究提出假设:

假设 H10：互联网辟谣平台用户功能满足度正向影响用户满意度

本研究针对互联网辟谣平台用户的使用意愿, 通过权衡需求理论引入功能满足变量, 在 社会交换理论的背景下引入感知个人收益及其影响因素，研究模型如图 1 所示。

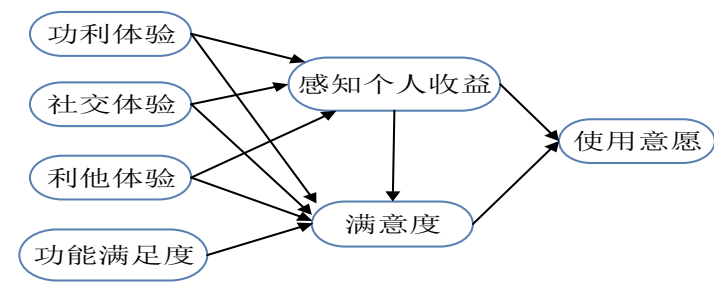

图 1 互联网辟谣平台用户使用意愿研究模型

\section{3. 研究方法与数据收集}

本研究样本数据的手机采用问卷调查的方式。首先, 需要进行测量量表的开发。为了有 较好的信度和效度，所有变量测量的设计均来源于国内外相关文献，并根据网络辟谣平台的 特征做相应调整。针对 7 个潜变量设计题项，测量题项采用李克特 5 级评分法。其次，在大 范围调查前, 先在小范围内进行预调查, 根据反馈意见修改和完善阅卷, 得到最终的正式调 查问卷,调查最终共获得 289 份有效问卷。

\section{4. 数据分析与结果}

本研究通过结构方程模型, 数据处理采用偏最小二乘法。从两个方面对模型进行验证: 测量模型验证和结构模型验证。

测量模型检验需要检验量表的信度和效度。通过 SmartPLS 计算结果显示, 各变量的组合 信度与克隆巴赫系数均大于 0.8 , 平均方差抽取量都大于 0.5 , 说明测量题项内部具有较好的 信度。效度是从收敛效度和区分效度两方面进行检验。通过 SmartPLS 软件计算变量的交叉 因子负荷都大于 0.7 , 说明收玫效度符合标准。见表 1 所示, 表格左对角线数据为平均方差抽 取量平方根，该数据大于所在行和列的所有数值，因此，测量模型的区分效度合格。

表 1 潜变量相关系数与 AVE 平方根

\begin{tabular}{cccccccc}
\hline \hline \multicolumn{2}{c}{ 测量变量 } & \multicolumn{5}{c}{ 相关系数矩阵 } \\
\hline & PI & AE & UE & SE & FS & SA & CI \\
PI & 0.873 & & & & & & \\
AE & 0.546 & 0.904 & & & & & \\
UE & 0.463 & 0.565 & 0.773 & & & & \\
SE & 0.582 & 0.467 & 0.526 & 0.862 & & & \\
FS & 0.348 & 0.615 & 0.547 & 0.318 & 0.812 & & \\
SA & 0.224 & 0.472 & 0.363 & 0.512 & 0.393 & 0.903 & \\
CI & 0.594 & 0.643 & 0.343 & 0.458 & 0.236 & 0.284 & 0.879
\end{tabular}
意愿

注: PI-感知个人收益, $\mathrm{AE}$-利他体验, UE-功利体验, SE-社交体验, FS-功能满足度, SA-满意度, $\mathrm{CI}$-使用

结构模型验证通过 Smart PLS 软件计算路径系数，模型验证结果显示，假设 H1-H10 均 得到支持。 


\section{5. 结语}

为讨论公众是否愿意使用互联网辟谣平台的影响因素, 本研究从感知个人收益和满意度 两方面提出了四个可能的因素, 构建假设模型、收集实证数据, 并用最小二乘法对数据进行 处理, 最终从测量模型和结构模型两方面对模型进行了验证。本研究也得到了一些有意义的 结论, 但仍存在局限性, 互联网辟谣平台的系统功能满足度对用户的满意度有直接影响, 但 是未说明平台系统的具体功能要素与用户满意度和使用意愿的影响以及影响程度如何, 以后 可针对互联网辟谣平台系统功能方面展开讨论。

\section{References}

[1] Zhai Yue, Xia Zhijie, Ge Tao. Exploring the construction of rumor information website to optimize user performance [j]. Library Science Research, 2015(16): 37-42.

[2] Yang Xiu.Review of the status quo and problems of local government's response to online rumors_-Based on the study of typical cases of "attacking Internet rumors"[J].Journal of Chongqing University(Social Science Edition),2016,22(06):175- 181.

[3] Huang Baizhen, Zhu Xiaodong. Research on the influencing factors of the willingness of mobile social app users to continue to use [j]. Modern Intelligence, 2016, 36 (12): 57-64.

[4] Li Wu, Hu Bo, Ji Dan. Research on User's Willingness to Use E-book Read Client_—Based on utaut and vam Theory [j]. Library Forum, 2018, 38(04): 103-110.

[5] Khan S A,Liang Y,School S.An Empirical Study of Perceived Factors Affecting Customer Satisfaction to Repurchase intention in Online Stores in China[J].Journal of Service Science and Management,2015,8(3):291-305.

[6] Butler B S.Membership Size,Communication Activity,and Sustainability:A Resource Based Model of Online Social Structures[R].Information Systems Research, 2001,12( 4):346-362.

[7] QU Wei, Zhang Huiying. Research on the Influence Mechanism of Continuous Use Intention of Users in Relational Virtual Community_-The Regulating Role of Interpersonal Trust[J].Journal of the China Society of Information Science,2016,35(04):415-424. 